\title{
Bulk tank milk surveillance as a measure to detect Coxiella burnetii shedding dairy goat herds in the Netherlands between 2009 and 2014
}

\author{
R. Van den Brom, ${ }^{* 1}$ I. Santman-Berends, $†$ S. Luttikholt, ${ }^{*}$ L. Moll, ${ }^{*}$ E. Van Engelen, $†$ and P. Vellema* \\ *Department of Small Ruminant Health, and \\ †Department of Research and Development, GD Animal Health, 7400 AA Deventer, the Netherlands
}

\begin{abstract}
In the period from 2005 to 2009, Coxiella burnetii was a cause of abortion waves at 28 dairy goat farms and 2 dairy sheep farms in the Netherlands. Two years after the first abortion waves, a large human Q fever outbreak started mainly in the same region, and aborting small ruminants were regarded as most probable source. To distinguish between infected and noninfected herds, a surveillance program started in October 2009, based on PCR testing of bulk tank milk (BTM) samples, which had never been described before. The aim of this study was to analyze the effectiveness of this surveillance program and to evaluate both the effect of culling of pregnant dairy goats on positive farms and of vaccination on BTM results. Bulk tank milk samples were tested for C. burnetii DNA using a real-time PCR, and results were analyzed in relation to vaccination, culling, and notifiable (officially reported to government) C. burnetii abortion records. In spring and autumn, BTM samples were also tested for antibodies using an ELISA, and results were evaluated in relation to the compulsory vaccination campaign. Between October 2009 and April 2014, 1,660 (5.6\%) out of 29,875 BTM samples from 401 dairy goat farms tested positive for $C$. burnetii DNA. The percentage of positive samples dropped from $20.5 \%$ in 2009 to $0.3 \%$ in 2014. In a multivariable model, significantly higher odds of being PCR positive in the BTM surveillance program were found in farms of which all pregnant dairy goats were culled. Additionally, the risk for $C$. burnetii BTM PCR positivity significantly decreased after multiple vaccinations. Bulk tank milk ELISA results were significantly higher after vaccination than before. The ELISA results were higher after multiple vaccinations compared with a single vaccination, and ELISA results on officially declared infected farms were significantly higher compared with noninfected farms. In conclusion, BTM surveillance is an effective and use-
\end{abstract}

Received October 28, 2014.

Accepted February 12, 2015

${ }^{1}$ Corresponding author: r.vd.brom@gdanimalhealth.com ful tool to detect $C$. burnetii shedding dairy goat herds and to monitor a $\mathrm{Q}$ fever outbreak, and thus the effect of implemented measures.

Key words: Coxiella burnetii, bulk tank milk, surveillance, dairy goat, polymerase chain reaction

\section{INTRODUCTION}

Q fever is a zoonosis caused by Coxiella burnetii, an aerobic, obligate intracellular, gram-negative, highly resistant bacterium that may infect mammals, birds, and arthropods (Babudieri and Moscovici, 1952; ArricauBouvery and Rodolakis, 2005; Berri et al., 2007). In infected domestic ruminants, the primary animal reservoir of $C$. burnetii, the main symptom is abortion; after abortion or parturition, shedding mainly takes place in birth products but also occurs in urine, feces, and milk (Arricau Bouvery et al., 2003; Guatteo et al., 2007; Rodolakis et al., 2007; García-Pérez et al., 2009; Roest et al., 2012).

In the period from 2005 to 2009, C. burnetii was demonstrated as the cause of abortion waves at 28 dairy goat farms and 2 dairy sheep farms in the Netherlands (Vellema and Van den Brom, 2014). In 2007, a large human Q fever outbreak started mainly in the same region where abortion waves occurred, and aborting small ruminants were increasingly regarded as the most probable source (Van Steenbergen et al., 2007; van der Hoek et al., 2012). Through April 2014, more than 4,200 human cases were officially reported (www.RIVM.nl).

In 2008, the second year of the outbreak, several measures on dairy sheep and dairy goat farms were implemented, aiming at preventing environmental contamination and human infections (Van den Brom and Vellema, 2009). However, when the number of human Q fever patients further increased, additional measures were implemented, and after a voluntary start at the end of 2008, compulsory vaccination of all dairy sheep and dairy goats was carried out in the outbreak area in 2009; from 2010 onward, in the whole country a phase 1 vaccine (Coxevac, CEVA Santé Animale, Libourne, France) was mandated, which was not licensed at that time and only limitedly available. To distinguish be- 
tween infected and noninfected herds, a surveillance program started in October 2009 based on PCR testing of bulk tank milk (BTM) samples. At the end of 2009, the government decided to cull all pregnant sheep and goats on officially declared $C$. burnetii BTM PCRpositive farms. For nonpregnant sheep and goats on C. burnetii BTM PCR-positive farms, a breeding ban for life was implemented. The aim of the current study was to analyze all results from BTM testing of dairy goat farms in the Dutch surveillance program and, retrospectively, (1) to determine the usability of BTM surveillance to detect $C$. burnetii shedding dairy goat herds, (2) to evaluate the effect of culling of pregnant dairy goats on $C$. burnetii BTM PCR positivity on initially positive farms, and (3) to evaluate the effect of vaccination.

\section{MATERIALS AND METHODS}

\section{Study Population and BTM Samples}

In 2008, on 392 professional dairy goat farms with more than 200 dairy goats per farm, approximately 260,000 goats were kept with an average of approximately 900 animals per farm (Van den Brom and Vellema, 2009). The majority of these farms were situated in the southeastern part of the Netherlands.

In autumn 2008, all 392 Dutch dairy goat farmers with more than 200 dairy goats per farm were requested to submit a BTM sample to be tested for C. burnetii, using a real-time PCR (LSI Taqvet $C$. burnetii, Laboratoire Service International, Lissieu, France), which targets the repetitive transposon-like region of the bacterium. The test was performed according to the manufacturer's instructions and results are presented in 3 classes: negative $(<100$ bacteria/mL), high positive ( $\leq 100$ to $<10,000$ bacteria/mL based on PCR), and very high positive (PCR $\geq 10,000$ bacteria/mL), as proposed by Van den Brom et al. (2012b). In October 2009, a surveillance program based on BTM testing became mandatory for all dairy sheep and dairy goat farms with more than 50 animals, and BTM samples were tested twice a month during the lambing season, between December 1 and June 30. Outside the lambing season, BTM samples were tested monthly on noninfected farms and twice a month on infected farms. In spring and autumn, BTM samples were also tested using an indirect ELISA (Ruminants Serum Q Fever LSI Kit, Laboratoire Service International) according to the manufacturers' instructions, and ELISA results were evaluated in relation to the compulsory vaccination, which has to be executed annually before the first of August (breeding season). The ELISA results were expressed by sample/positive (SP) percentages, calcu- lated using the formula: $\left(\mathrm{OD}_{\text {sample }}-\mathrm{OD}\right.$ negative control $) /$ $\left(\mathrm{OD}_{\text {positive control }}-\mathrm{OD}\right.$ negative control $) \times 100 \%$, where OD = optical density.

\section{Officially Declared C. burnetii-Infected Herds}

According to legislation, C. burnetii BTM PCR-positive results at GD Animal Health had to be confirmed by the national reference laboratory (Central Veterinary Institute, CVI, Lelystad, the Netherlands); after confirmation, an official BTM sample was collected by the Dutch Food and Consumer Product Safety Authority (Utrecht, the Netherlands) to be tested by CVI. When the official BTM sample was found positive, a farm was officially declared $C$. burnetii positive.

\section{Farms with a History of C. burnetii Abortion}

Coxiella burnetii was first diagnosed as the cause of abortion waves on 2 dairy goat farms in 2005 and confirmed by immunohistochemistry in sections of fetal membranes. Immunohistochemistry was performed using the EnVision+ system (DAKO Corporation, Carpinteria, CA; Wouda and Dercksen, 2007; Van den Brom et al., 2012a). Between 2005 and 2008, C. burnetii was detected in placental membranes of aborted fetuses submitted from 15 dairy goat farms. In the Netherlands, small ruminant abortion rates exceeding $5 \%$ became notifiable in 2008. From that moment onwards, in 2008 and 2009, on 11 dairy goat farms that reported abortion problems, C. burnetii was confirmed as the cause (Van den Brom et al., 2012a).

\section{Vaccination Records}

In the Netherlands, a voluntary vaccination campaign against $C$. burnetii with a phase 1 vaccine (Coxevac, CEVA Santé Animale) started in 2008. At that time, because of a lack of vaccine, voluntary vaccination was only carried out on 26 dairy goat farms located within a radius of $45 \mathrm{~km}$ around the village of Uden, the center of the human Q fever outbreak. In 2008, approximately 40,000 dairy goats were voluntarily vaccinated. In 2009, vaccination of all dairy goats was mandatory in the high incidence area, the area within a $45-\mathrm{km}$ radius around the village of Uden, completed with the rest of the province of Noord-Brabant, and voluntary vaccination was possible for a limited number of farms outside the high incidence area. In 2009, goats on 142 farms were compulsorily or voluntarily vaccinated. Since 2010 , vaccination of all dairy goats has been mandatory in the whole country. The vaccinations have to be executed annually before August 1 or after animals reach the age of 3 mo. Date of vaccination of each individual goat 
is compulsorily registered in the national identification and registration database for small ruminants and were available for our study. For calculation purposes, the date of vaccination of the lactating goats was used.

\section{Statistical Analysis}

For analytical purposes, all data were combined and checked. Farms that appeared to keep sheep instead of goats during a part of the analyzed period were removed from the data set $(n=8)$. On religious grounds, 2 dairy goat farmers requested and were exempted from compulsory vaccination; data from these farms were excluded as well.

Two different data sets were composed. The first data set contained combined results of 2-weekly or 4-weekly BTM PCR results combined with results from farms with abortion waves, officially infected farms, and vaccination data. The complete data set consisted of 29,875 PCR results obtained between October 2009 and May 2014 from 401 goat farms. For descriptive purposes, all observations were included. For analytical purposes, only observations of PCR results between July 2010 and the end of 2013 were included. Observations before July 2010 were not included in the analyses because before this date interventions took place on farms that tested PCR positive, including the culling of pregnant goats.

The second data set contained ELISA results combined with results from farms with abortion waves and from infected herds and vaccination data. After removing double observations and ELISA results that were not obtained as part of the BTM surveillance program, 3,006 observations remained from in total 401 different dairy goat farms. In addition, for the analyses of ELISA results, the period from the start of the BTM surveillance program until the end of 2013 remained for analytical purposes, and eventually 2,703 observations were included.

Regarding both data sets, descriptive results are presented comparing the period before and after vaccination and before and after culling using STATA 13 (Stata Corp. LP, College Station, TX). Percentage of goat farms with a PCR-positive result are presented for all dairy goat farms together and for 3 subgroups of dairy farms:

1. Farms that were officially declared to be infected between October 2009 and July 2010 versus the remaining dairy goat farms;

2. Farms with a history of $C$. burnetii abortions versus the remaining dairy goat farms;

3. Farms that tested negative 3 times versus farms that tested positive $(>100)$ or farms that tested high positive $(>1,000)$ in at least 1 of the first 3 mo of the surveillance program in 2009.

ELISA results were normally distributed and therefore means and confidence intervals were used for descriptive purposes.

To evaluate the effect of culling and vaccination on PCR or ELISA results, multivariable multilevel linear and logistic analyses were performed (mixed and melogit in STATA). The PCR result (binary variable) and ELISA SP value (continuous variable) were included as dependent variable in the models. Variables that were included as independent variables were measurements prior versus measurements postvaccination within a year (both models), number of years a herd was vaccinated (both models), farms that were officially declared to be infected versus all other farms (both models), and month of sampling (PCR). By using multilevel models, the model was corrected for the fact that BTM results within the same herd were more comparable than BTM results between different farms. The best model was evaluated by comparing the log-likelihood between the full and the nested model using the likelihood ratio test. Residuals of the models were monitored to evaluate whether models that were used met the assumptions of linear and logistic regressions, and the model fit was evaluated using the $\mathrm{R}^{2}$.

\section{RESULTS}

\section{BTM PCR Results}

Descriptive Results. Between October 2009 and April 2014, 29,875 BTM samples from 401 dairy goat farms were tested for C. burnetii by PCR, and 1,660 $(5.6 \%)$ of these samples from 142 different farms tested positive (Figure 1). The majority of positive samples was found in 2009 when, in the 3 rounds performed, on average $20.5 \%$ of the samples tested positive; this declined from $25 \%$ in October to $15 \%$ in December, and declined again to $0.3 \%$ in 2014 (Figure 2). Descriptive results of $C$. burnetii infections in the Netherlands between 2005 and April 2014 are presented in Table 1.

Not all farms participated in all rounds of the mandatory BTM surveillance program because of various reasons (e.g., some farmers started farming and others stopped farming during the surveillance period, and on some farms all adult goats did have a dry period). From 355 dairy goat farms at least 25 samples per farm were tested and, out of these, 222 farms (63\%) always tested negative, 64 farms (18\%) tested positive 1 to 5 times, 21 farms $(6 \%)$ tested positive 6 to 10 times, 25 farms (7\%) tested positive 11 to 20 times, and 23 farms $(6 \%)$ tested positive more than 20 times. Based on BTM 


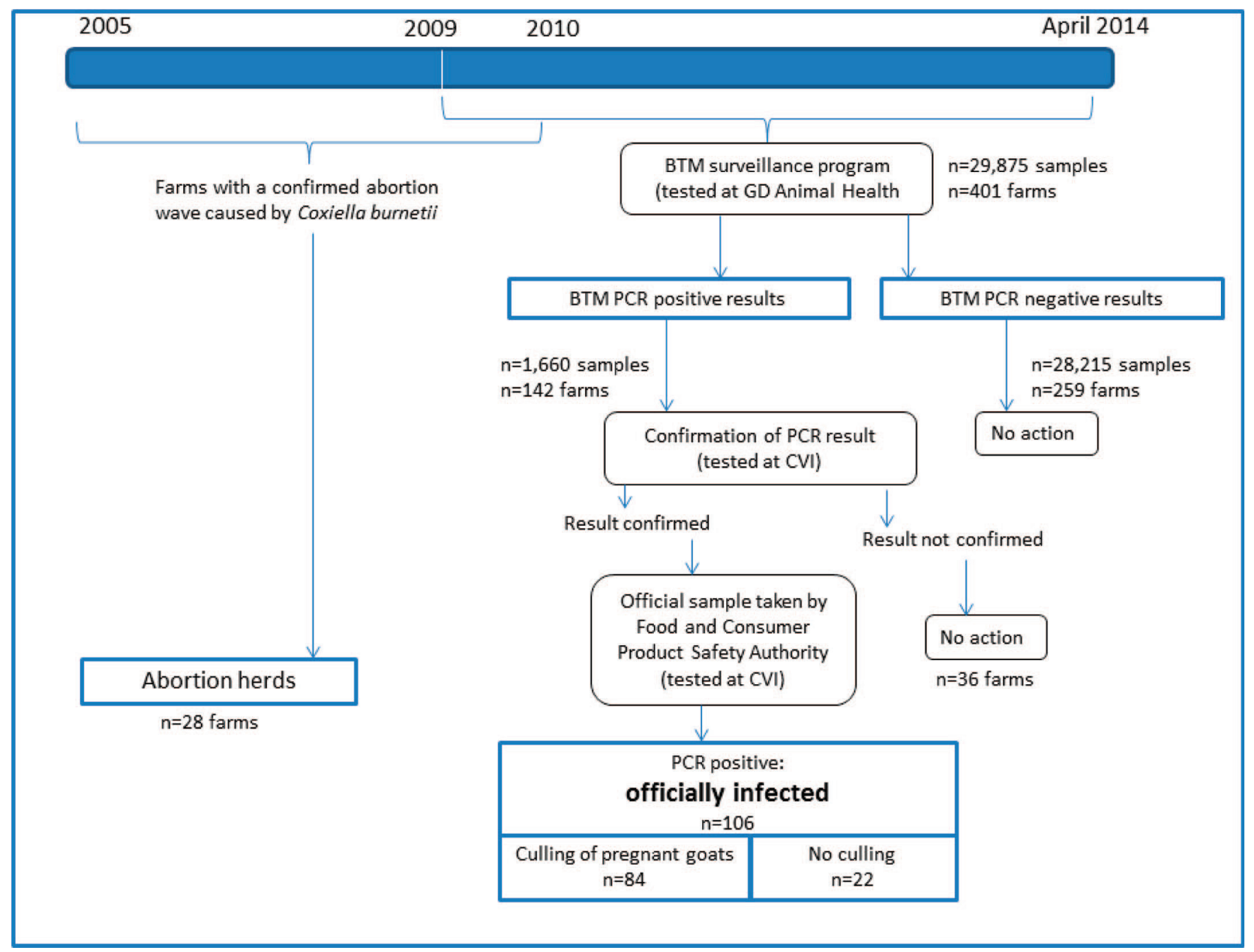

Figure 1. Bulk tank milk surveillance results in diagram form. Abortion waves caused by Coxiella burnetii were confirmed on 28 dairy goat farms between 2005 and 2010. Between October 2009 and April 2014, 29,875 bulk tank milk (BTM) samples from 401 dairy goat farms were tested by PCR at GD Animal Health. Samples that tested positive (1,660 from 142 farms) were submitted to the Central Veterinary Institute (CVI; Lelystad, the Netherlands), and after confirmation the Dutch Food and Consumer Product Safety Authority (NVWA; Utrecht, the Netherlands) collected an official BTM sample which was tested by PCR at CVI. If this official BTM sample tested positive, a farm was declared officially infected $(\mathrm{n}=106)$. Between October 2009 and July 2010, pregnant dairy goats on 84 officially declared infected farms were culled. After July 2010, culling was not applicable for officially declared infected farms $(\mathrm{n}=22)$. Color version available online.

PCR results, 106 dairy goat farms $(67,33,4$, and 2 in 2009, 2010, 2011, and 2012, respectively) were officially declared infected (Figure 1).

The BTM PCR results are presented for the entire group of goat farms and for stratified farms based on (1) BTM results during the first 3 rounds of the surveillance program, (2) whether or not pregnant goats were culled, and (3) abortion history. The different groups of goat farms are schematically presented in Figure 1. Figure 2 shows the development of the percentage of dairy goat farms with a $C$. burnetii BTM PCR-positive result during the surveillance period. In the first 3 rounds of the BTM surveillance program in $2009,28 \%$ of all dairy goat farms tested BTM PCR positive in at least 1 of the rounds. In both groups with positive results, the percentage of PCR-positive farms decreased in time (Figure 3a). On 84 dairy goat farms that were officially declared infected between October 2009 and July 2010, all pregnant animals were culled. The progress of the BTM PCR results in time is presented in Figure 3b.

BTM PCR Results on Farms with a History of Abortion Caused by C. burnetii. Out of the 28 dairy goat farms with an abortion wave between 2005 and 2010, 24 participated in the mandatory BTM surveillance (Table 2). On 1 farm, 2 C. burnetii abortion waves were confirmed-1 in 2006 and another in 
Table 1. Descriptive results of Coxiella burnetii infections in the Netherlands between 2005 and April $2014^{1}$

\begin{tabular}{|c|c|c|c|c|c|c|c|c|c|c|}
\hline Item & 2005 & 2006 & 2007 & 2008 & 2009 & 2010 & 2011 & 2012 & 2013 & 2014 \\
\hline No. of farms with $C$. burnetii abortion & & 6 & 7 & 7 & 6 & 0 & 0 & 0 & 0 & 0 \\
\hline $\begin{array}{l}\text { No. (\%) participating in bulk tank milk (BTM) } \\
\text { sampling }\end{array}$ & $\mathrm{NA}^{2}$ & NA & NA & $\begin{array}{l}292 \\
(74.5)\end{array}$ & $\begin{array}{c}392 \\
(100)\end{array}$ & $\begin{array}{c}375 \\
(100)\end{array}$ & $\begin{array}{c}348 \\
(100)\end{array}$ & $\begin{array}{c}343 \\
(100)\end{array}$ & $\begin{array}{c}336 \\
(100)\end{array}$ & $\begin{array}{c}332 \\
(100)\end{array}$ \\
\hline No. (\%) of newly BTM PCR-positive farms & NA & NA & NA & $\begin{array}{c}66 \\
(32.9)\end{array}$ & $\begin{array}{c}51 \\
(13.0)\end{array}$ & 30 & $\begin{array}{l}7 \\
(2.0)\end{array}$ & $\begin{array}{l}1 \\
(0.3)\end{array}$ & $\begin{array}{l}0 \\
(0.0)\end{array}$ & $\begin{array}{l}1 \\
(0.3)\end{array}$ \\
\hline $\begin{array}{l}\text { No. (\%) of repeatedly BTM PCR-positive } \\
\text { farms }\end{array}$ & NA & NA & NA & NA & $\begin{array}{l}52 \\
(78.8)\end{array}$ & $\begin{array}{l}81 \\
(69.2)\end{array}$ & $\begin{array}{c}59 \\
(40.1)\end{array}$ & $\begin{array}{l}36 \\
(23.4)\end{array}$ & $\begin{array}{c}21 \\
(13.5)\end{array}$ & $\begin{array}{l}5 \\
(3.2)\end{array}$ \\
\hline No. (\%) of repeatedly officially infected farms & NA & NA & NA & NA & NA & $(0.0)$ & $(7.0)$ & $(3.8)$ & $\begin{array}{l}4 \\
(3.8)\end{array}$ & $\begin{array}{l}0 \\
(0.0)\end{array}$ \\
\hline No. (\%) of vaccinations ${ }^{3}$ & NA & NA & NA & $\begin{array}{l}26 \\
(6.6)\end{array}$ & $\begin{array}{l}142 \\
(36.2)\end{array}$ & $\begin{array}{c}375 \\
(100)\end{array}$ & $\begin{array}{c}348 \\
(100)\end{array}$ & $\begin{array}{c}343 \\
(100)\end{array}$ & $\begin{array}{c}336 \\
(100)\end{array}$ & $\begin{array}{c}332 \\
(100)\end{array}$ \\
\hline Notifiable human $\mathrm{Q}$ fever patients & $17^{4}$ & $17^{4}$ & 168 & 1,000 & 2,354 & 504 & 81 & 66 & 19 & $19^{5}$ \\
\hline
\end{tabular}

${ }^{1}$ Between 2005 and 2008, there were approximately 350 dairy goat farms in the Netherlands. From 2009 on, exact numbers are known. Between 2005 and 2010, abortion storms caused by C. burnetii were detected 28 times on dairy goat farms. In 2008, BTM samples were tested by real-time PCR for the first time on a voluntary basis. From 2009 on, all dairy goats farms were mandatory tested using a C. burnetii BTM PCR. A total of 106 dairy goat farms were officially declared infected. Culling of pregnant animals on C. burnetii BTM PCR-positive farms was performed between December 2009 and July 2010. Vaccination against C. burnetii started in 2008, was intensified in 2009, and became mandatory nationwide in 2010. The number of officially reported human Q fever patients between 2007 and August 27, 2014, was 4,211.

${ }^{2} \mathrm{NA}=$ not applicable.

${ }^{3}$ Farms with religious reasons not to vaccinate $(\mathrm{n}=2)$ were excluded from the data set.

${ }^{4}$ Annually, an average of 17 human Q fever cases occurred between 1978 and 2006.

${ }^{5}$ Until August 27, 2014.

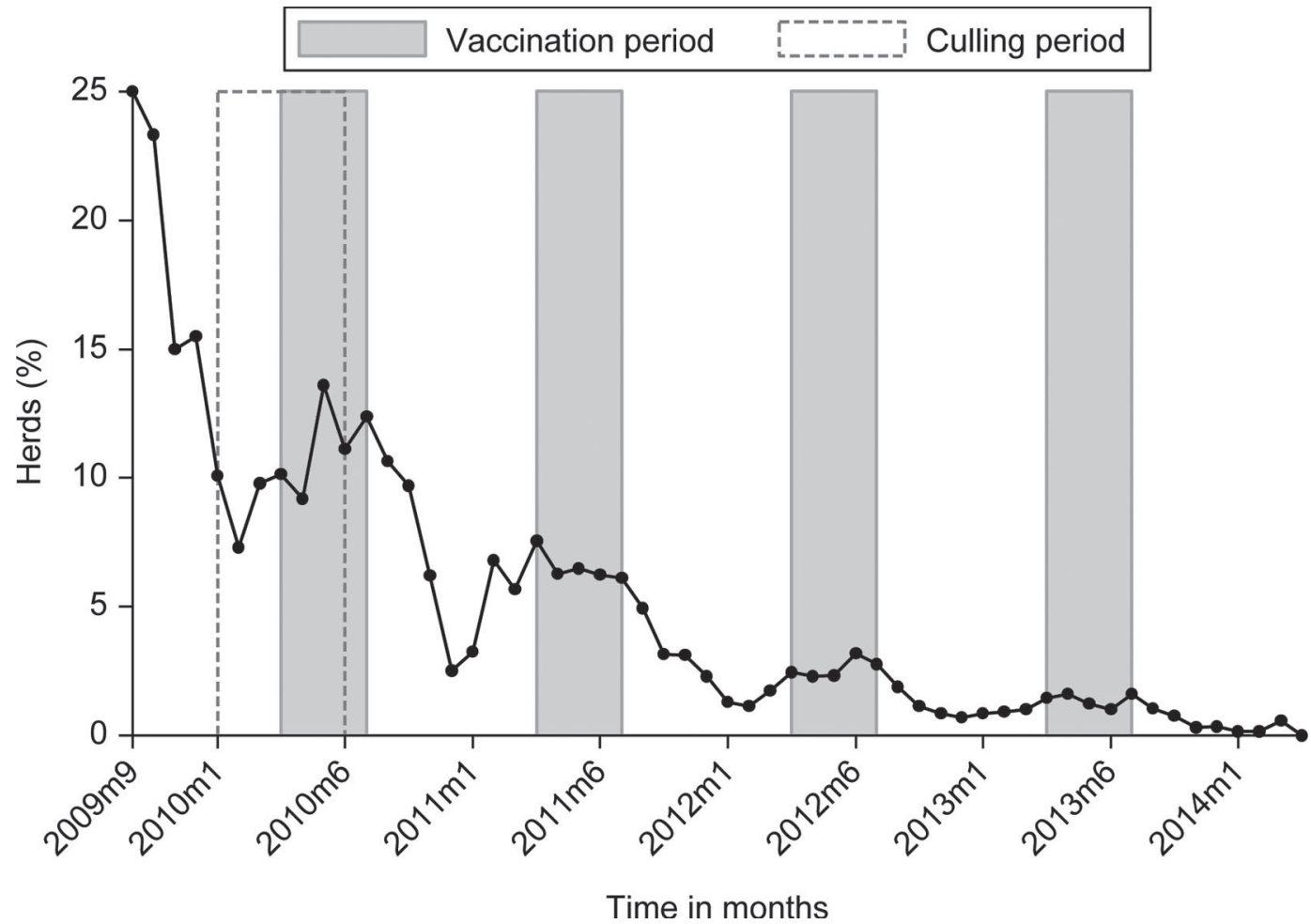

Figure 2. Percentage of Dutch dairy goat farms with a Coxiella burnetii bulk tank milk (BTM) PCR-positive result ( $>100$ bacteria/mL) between October 2009 (2009m9) and April 2014. After a voluntary start in October 2008, and a partly mandatory vaccination in 2009, mandatory vaccination is executed on all dairy goat farms annually before the first of August since 2010. Vaccination is carried out between April and August (vaccination period). Between December 2009 and July 2010, pregnant dairy goats were culled (culling period) on all officially declared C. burnetii PCR-positive farms. 
a)

\begin{tabular}{|c|c|}
\hline Max. BTM result in 2009 $=>1000(n=64)$ & Culling period \\
\hline Max. BTM result in 2009 $=>100 \&<1000(n=39)$ & Vaccination period \\
\hline Max. BTM result in $2009<100(n=265)$ & \\
\hline
\end{tabular}

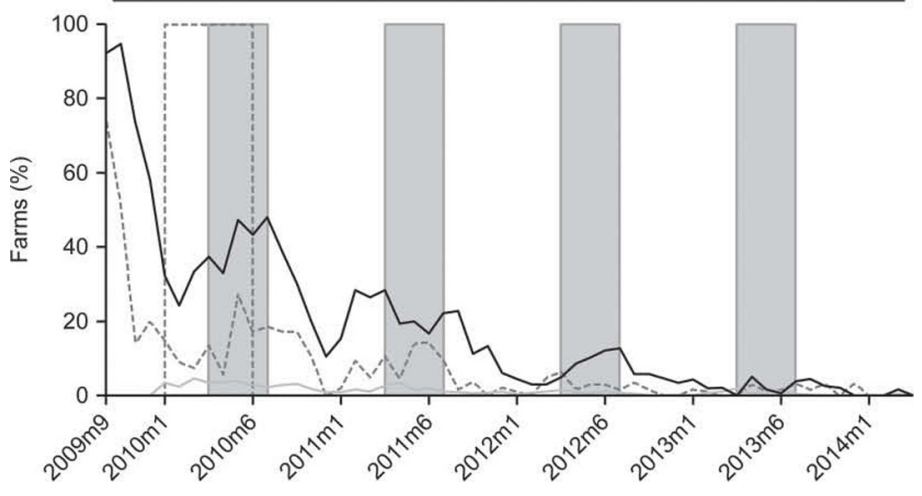

Time in months
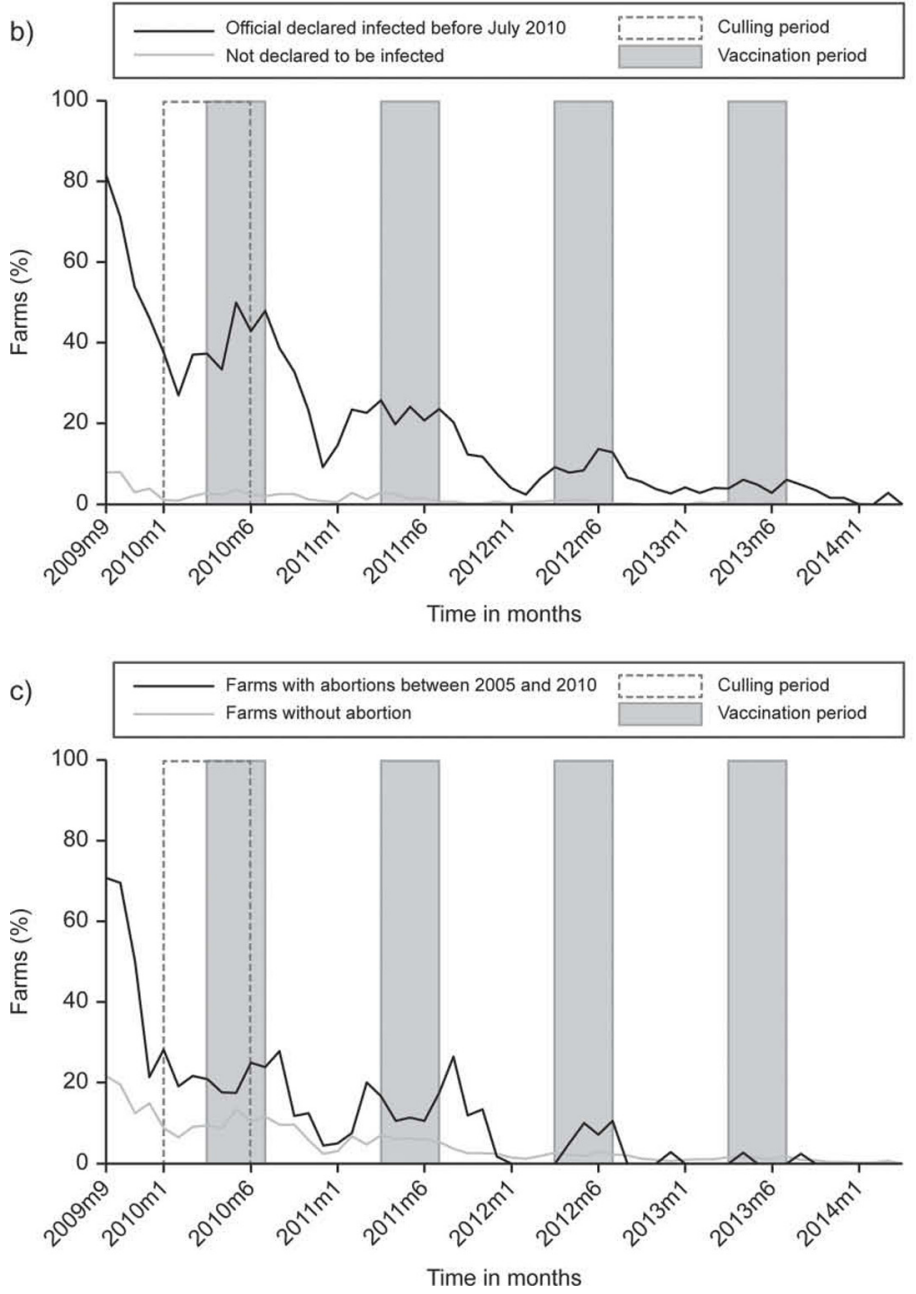

Figure 3. (a) Percentage of Dutch dairy goats farms with a Coxiella burnetii bulk tank milk (BTM) PCR-positive result (>100 bacteria/ $\mathrm{mL}$ ), divided into 3 groups based on their highest $C$. burnetii BTM PCR result in 2009. (b) Percentage of Dutch dairy goats farms with a $C$. burnetii BTM PCR-positive result (>100 bacteria/mL), divided into 2 groups, an officially declared $C$. burnetii infected and a noninfected group. (c) Percentage of Dutch dairy goats farms with a C. burnetii BTM PCR-positive result (>100 bacteria/mL), divided into 2 groups, farms with abortion caused by C. burnetii and farms without officially reported abortion caused by C. burnetii. After a voluntary start in October 2008, and a partly mandatory vaccination in 2009, mandatory vaccination is executed on all dairy goat farms annually before the first of August since 2010. Vaccination is carried out between April and August (vaccination period). Between December 2009 and July 2010 , pregnant dairy goats were culled (culling period) on all officially declared C. burnetii PCR-positive farms. $\mathrm{m}=$ month. 
2009 - and the 3 remaining farms stopped farming before the start of the compulsory BTM surveillance program. Based on their BTM PCR results, 13 out of the 24 participating farms were officially declared infected in 2009 or 2010. Alterations in BTM results from these farms in time are presented in Figure 3c. Dairy goat farms with an abortion wave confirmed to be caused by C. burnetii were significantly $(P<0.001)$ more likely to be officially declared $C$. burnetii-infected based on BTM. In addition, farms with an abortion wave caused by $C$. burnetii in more recent years (2008 or 2009) were significantly more often declared $C$. burnetii infected compared with farms with an abortion wave between 2005 and $2007(P=0.02)$.

Multivariable Analysis. In a multivariable model in which all observations of the BTM surveillance pro-

Table 2. Descriptive information on dairy goat farms with an abortion storm caused by Coxiella burnetii

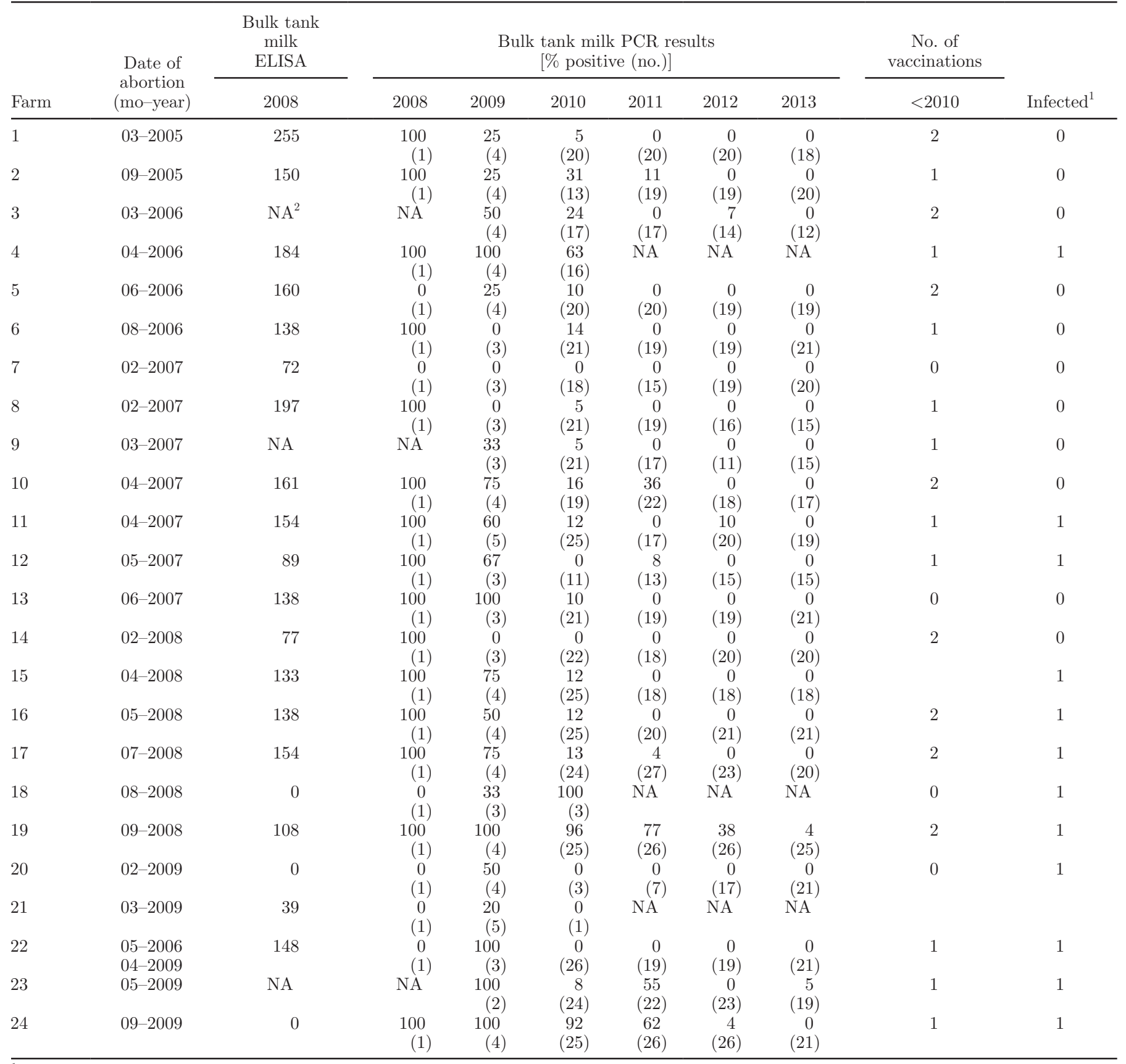

${ }^{1}$ Officially declared C. burnetii-positive based on BTM PCR.

${ }^{2} \mathrm{NA}=$ not applicable. 
gram between July 2010 and April 2014 were included, significantly higher odds of testing BTM PCR positive were found in the months February until November, varying between 1.7 and 8.5, compared with January. The odds increased during the lambing season between January and April and increased even further with the highest odds in July, August, and September. Most vaccinations were performed in July. The odds of testing PCR positive after the culling period, between July 2010 and April 2014, was significantly higher in farms of which all pregnant dairy goats were culled compared with farms which were not officially declared infected during the culling period. Finally, the risk for C. burnetii BTM PCR positivity significantly decreased after multiple years of vaccination (Table 3 ).

\section{BTM ELISA Results}

A total of 3,006 BTM ELISA results were available from 401 dairy goat farms. Average ELISA results before and after vaccination, both for officially declared infected and noninfected farms, are presented in Figure 4. Average BTM ELISA results on officially infected dairy goat farms were consistently high positive, even before first vaccination. In noninfected dairy goat herds, average BTM ELISA results increased more gradually until the second vaccination, and remained high positive thereafter. Average BTM ELISA results before the first vaccination were significantly higher in officially infected dairy goat herds compared with noninfected herds (estimate $=140 ; 95 \% \mathrm{CI}=120-161$ ). Because the progression in SP ELISA values evolved differently in officially infected and noninfected herds, a stratified analysis on type of herd was performed. In the multivariable model for officially infected dairy goat herds, BTM ELISA results were significantly higher after vaccination compared with before vaccination (30; 95\% CI $=19-41)$. After the first vaccination, the average ELISA results remained stable at a high level and did not significantly increase further after following multiple vaccinations. In the multivariable model for noninfected dairy goat herds, BTM ELISA results were significantly higher after compared with before vaccination as well $(24 ; 95 \% \mathrm{CI}=18-29)$. In addition, after every next vaccination, ELISA results increased further to a high and stable level after 5 vaccinations (Table 4).

\section{DISCUSSION}

The human Q fever outbreaks in 2007 and 2008 stimulated the start of a large multidisciplinary research portfolio aiming at generating knowledge to take adequate control measures (Van den Brom and Vellema, 2009). Voluntarily submitted BTM samples from dairy goat farms were tested by PCR and ELISA in 2008 and 2009 as part of this research portfolio, although at that time it was unknown how to interpret the results. After comparing BTM PCR and ELISA results with the serological status of 13 animals per farm, and determining correlations with a farm history

Table 3. Final results of a multivariable model for risk factors associated with Coxiella burnetii bulk tank milk (BTM) PCR-positive results

\begin{tabular}{llcrcc}
\hline \multirow{2}{*}{ Item } & Subset & $\begin{array}{c}\text { Odds } \\
\text { ratio }\end{array}$ & $95 \%$ CI & $P$-value \\
\hline Month & January & 1.0 & & & \\
& February & 1.7 & 1.1 & 2.8 & 0.02 \\
& March & 1.8 & 1.1 & 2.8 & 0.02 \\
& April & 2.7 & 1.7 & 4.3 & $<0.001$ \\
& May & 2.9 & 1.9 & 4.6 & $<0.001$ \\
& June & 3.2 & 2.0 & 5.1 & $<0.001$ \\
& July & 4.8 & 3.2 & 7.3 & $<0.001$ \\
& August & 8.5 & 5.6 & 12.8 & $<0.001$ \\
& September & 6.3 & 4.1 & 9.8 & $<0.001$ \\
& October & 4.1 & 2.7 & 6.4 & $<0.001$ \\
Number of years the herd is vaccinated & November & 2.5 & 1.6 & 4.0 & $<0.001$ \\
& December & 0.9 & 0.5 & 1.4 & 0.57 \\
& 1 & 1.0 & & & \\
& 2 & 0.3 & 0.3 & 0.5 & $<0.001$ \\
& 3 & 0.1 & 0.1 & 0.2 & $<0.001$ \\
& 4 & 0.03 & 0.02 & 0.05 & $<0.001$ \\
Infected $^{2}$ & 5 & 0.01 & $<0.01$ & 0.02 & $<0.001$ \\
& & $<0.01$ & $<0.01$ & $<0.01$ & $<0.001$ \\
& No & 1.0 & & 576.2 & $<0.001$ \\
\hline
\end{tabular}

${ }^{1}$ Constant, probability of a dairy goat farm to have a $C$. burnetii BTM PCR-positive result.

${ }^{2}$ Officially declared C. burnetii positive based on BTM PCR. 
Table 4. Final results of a multivariable model for risk factors associated with Coxiella burnetii bulk tank milk (BTM) ELISA results

\begin{tabular}{|c|c|c|c|c|c|}
\hline Item & Subset & \multicolumn{2}{|c|}{ Officially infected herds } & \multicolumn{2}{|c|}{ Noninfected herds } \\
\hline Before vaccination & & Reference & & Reference & \\
\hline After vaccination & & $\begin{array}{l}29.7 \\
(18.8-40.6)\end{array}$ & $<0.001$ & $\begin{array}{l}23.7 \\
(18.4-29.1)\end{array}$ & $<0.001$ \\
\hline & 2 & $\begin{array}{l}57.7 \\
(35.1-80.3)\end{array}$ & $<0.001$ & $\begin{array}{l}196.1 \\
(187.3-205.0)\end{array}$ & $<0.001$ \\
\hline & 3 & $\begin{array}{l}40.7 \\
(17.8-63.3)\end{array}$ & $<0.001$ & $\begin{array}{l}190.5 \\
(181.7-199.4)\end{array}$ & $<0.001$ \\
\hline & 4 & $\begin{array}{l}36.4 \\
(12.8-60.1)\end{array}$ & 0.003 & $\begin{array}{l}223.5 \\
(213.6-233.5)\end{array}$ & $<0.001$ \\
\hline
\end{tabular}

${ }^{1}$ Average BTM ELISA result on a not officially C. burnetii declared infected dairy goat farm before the first vaccination.

of abortion caused by $C$. burnetii, the highest area under the receiver operator curve of the ELISA was at a PCR cutoff of 100 bacteria/mL (Van den Brom et al., 2012b), and this value was used during the BTM surveillance program which started in October 2009.
At that moment, there was no experience with BTM surveillance programs for $C$. burnetii. Initially, the aim of the BTM surveillance program in the Netherlands was to declare dairy goat farms $C$. burnetii-free after at least $1 \mathrm{yr}$ of BTM PCR-negative results. However, in

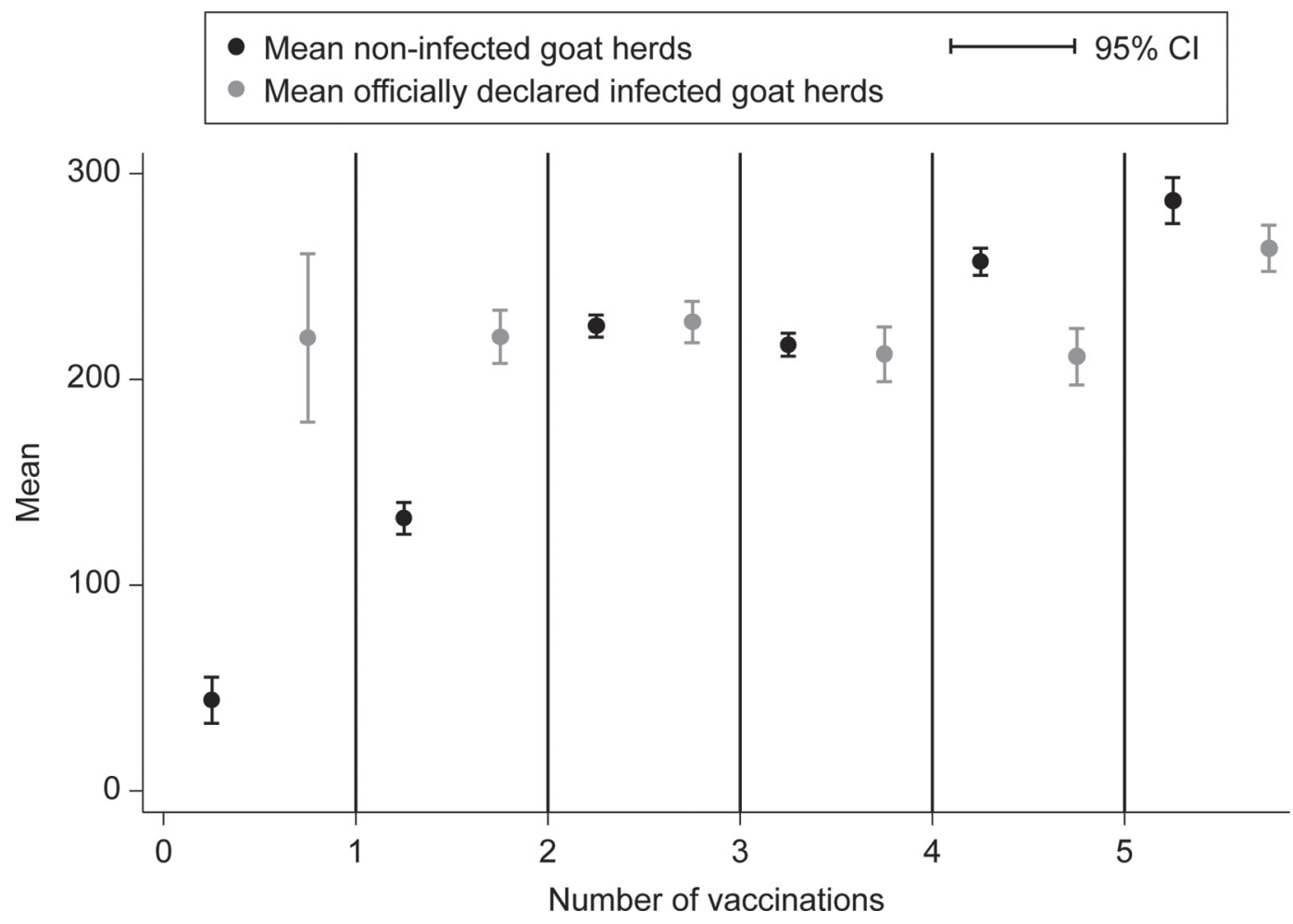

Figure 4. Average ELISA optical density results before and after vaccination, both for officially declared infected and noninfected dairy goat herds. 
December 2009, it was decided to interpret nonnegative results as positive results and these results were used to declare herds as C. burnetii infected. Following this decision, additional control measures, such as culling of all pregnant dairy goats and a lifetime breeding ban for the remaining goats in officially declared infected herds were implemented. Keeping this history in mind, it seemed worthwhile to retrospectively analyze the BTM surveillance program to determine its usability to detect $C$. burnetii shedding in dairy goat herds and, additionally, to evaluate implemented measures, such as culling of pregnant goats and vaccination.

In this retrospective cohort study, $28 \%$ of all dairy goat farms tested $C$. burnetii BTM PCR positive in at least 1 of the first 3 rounds of the surveillance program in 2009. Compared with 2009, a slightly higher proportion of dairy goat farms was BTM PCR positive in 2008 (32.9\%; 95\% CI $=30.2-35.6)$, although that prevalence was measured in voluntarily submitted BTM samples from $74.4 \%$ of the Dutch dairy goat farms (Van den Brom et al., 2012b). As surveillance programs for $C$. burnetii based on BTM samples from dairy goat farms are a relatively new phenomenon, only a small number of studies from other countries described results of $C$. burnetii BTM testing. In these countries (Fretz et al., 2007; Rahimi et al., 2011; Kampen et al., 2012; Boarbi et al., 2014), lower prevalence was found compared with the Netherlands at the start of the surveillance program. However, results of studies from other countries are difficult to compare with our results because of different study designs and the use of tests with different characteristics. To the best of our knowledge, the Netherlands was the first country where a C. burnetii BTM surveillance program was implemented aiming at distinguishing between infected and noninfected dairy goat farms at a nationwide level.

The odds of becoming BTM PCR positive differed throughout the year. Compared with January, significantly higher odds were found from February until November, with a pattern of a steady increase up to 8.5 in August followed by a decline to 2.5 in November and 0.9 in December. A possible explanation for the observed difference in risk in time could be the phenomenon that Dutch dairy goats are seasonal breeders (Van den Brom et al., 2013b). This seasonality of sexual activity is mainly influenced by day length, breed, and feeding; however, introduction of bucks in the herd, stress, or other unknown factors can also alter this seasonality, but this is hard to quantify. The breeding season starts in July, and after a gestation period of $5 \mathrm{mo}$, kidding starts on most farms not earlier than January and may last until June. In infected goats, a massive multiplication of $C$. burnetii can take place at the end of pregnancy (Sánchez et al., 2006) leading to shedding at parturition and afterward (Roest et al., 2012).

After a voluntary start in 2008 , compulsory vaccination started in the high incidence area in 2009, and has been mandatory since 2010 for all dairy goats in the Netherlands. This vaccination has to be done annually before the first of August. Vaccination not only influenced body temperature, but also gave locally adverse reactions at the vaccination site, a variable reduction in milk yield (Vellema et al., 2010a), and could act as stress factor, possibly affecting luteal function (Hesselink, 1993) and nonspecific immune response (Sejian and Srivastava, 2010). In 2010, it was shown that up to $9 \mathrm{~d}$ after vaccination with Coxevac, vaccine-derived C. burnetii DNA could be detected in individual milk samples from dairy goats (Hermans et al., 2011); in the discussion of their paper, those authors emphasize that a larger study was required to evaluate the effect of vaccination on BTM PCR results, suggesting that BTM could also become $C$. burnetii PCR positive by vaccination itself. However, these results could not be repeated on 4 farms where milk samples from 20 dairy goats per farm were individually repeatedly tested after vaccination using the same PCR test with the same cutoff as used for the mandatory $C$. burnetii BTM surveillance program. Additionally, in BTM samples from these 4 farms, no increased PCR results could be detected in the weeks following vaccination (Vellema et al., 2010b). The higher odds to become C. burnetii BTM PCR positive during the breeding season could indicate the possibility of shedding of $C$. burnetii during estrus, as has been described for Chlamydia abortus, another intracellular abortifacient agent in small ruminants (Papp et al., 1994; Livingstone et al., 2009).

Our results also show that the probability to become C. burnetii BTM PCR positive significantly decreases with increasing numbers of vaccinations. Vaccination seems very effective, especially when administered before the first pregnancy (Hogerwerf et al., 2011); because all dairy goats have been mandatorily vaccinated since 2010, this decrease was expected. Vaccinated, already chronically infected goats can intermittently shed $C$. burnetii, sometimes without a known trigger (Van den Brom et al., 2013b). Shedding by these goats probably mainly occurs in milk (Van den Brom et al., 2013b), but could also take place in birth products. Because of the lifetime breeding ban for the remaining goats in infected herds, massive shedding during parturition or abortion, and subsequent environmental contamination, was not likely to occur anymore. Presence of chronically infected goats on officially declared C. burnetii BTM PCR-positive farms can also explain why these farms are significantly more likely to have 
a positive BTM PCR result in the observed period. Although, in a herd with a mandatory lifetime breeding ban individually shedding dairy goats probably do not pose a risk for public health, they can be responsible for persistence of the infection within the herd. Therefore, detecting and removal of individual shedders as described previously (Rodolakis et al., 2007; Van den Brom et al., 2013b) should be considered on BTM PCR-positive farms.

The reduction in the number of $C$. burnetii BTM PCR-positive farms is most probably a consequence of the package of implemented measures, such as vaccination, culling, and a lifetime breeding ban. Bulk tank milk surveillance is an effective and useful tool to detect C. burnetii-shedding dairy goat herds and to monitor a Q fever outbreak, and thus the effect of implemented measures.

Farms with an abortion wave in 2008 and 2009 were more likely to become officially declared infected compared with farms with an abortion wave before 2008 . In our study, it was not possible to assess the cause of this. Both natural immunization, as has been described for Chlamydia abortus (Longbottom et al., 2013), and repeated vaccination could have played a role. In the Netherlands, Q fever in small ruminants is still a notifiable disease, and since the start of the mandatory vaccination program in 2010, no abortion caused by C. burnetii has been detected in small ruminants (van Engelen et al., 2014).

In 2009, the percentage of BTM-positive results on farms without reported $C$. burnetii abortion waves was relatively high. An explanation for this could be that not all infections with $C$. burnetii will lead to abortion waves. Massive shedding of $C$. burnetii at parturition is the main source of environmental contamination. High incidences of human $Q$ fever patients have been described around farms with officially reported abortion compared with BTM PCR-positive farms without reported abortion (van der Hoek et al., 2011, 2012).

Before the start of the vaccination campaign in 2008, BTM ELISA results showed that $87(29.8 \%)$ out of 292 voluntarily submitted BTM samples from dairy goat farms were serologically positive (Van den Brom et al., 2012b). Our study shows that within a year after vaccination BTM ELISA results were significantly higher compared with the period before. In addition, BTM ELISA results were significantly higher in officially infected dairy goat herds compared with noninfected herds. In a Belgian study, no significant difference was found in ELISA BTM results before and after vaccination on positive farms (Boarbi et al., 2014). Also, in our study, officially infected dairy goat herds were consistently high positive, although an increase in average BTM ELISA values was found after the first vac- cination. Thereafter, ELISA results in officially infected herds did not increase significantly anymore with more vaccinations performed, which is consistent with the results of Boarbi et al. (2014). In noninfected herds, BTM ELISA results increased when the herd was vaccinated more times. After noninfected herds had been vaccinated 2 times, BTM ELISA results were no longer significantly different from BTM ELISA results in officially declared infected herds (results not shown). In the current study, we did not analyze animal movements between farms as a possible source of infection, because officially declared infected farms were not allowed to sell goats with a breeding ban for purposes other than slaughter, and yearlings could only be sold when vaccinated before their first pregnancy on the farm where they were born.

In conclusion, BTM testing is an effective and useful tool to detect $C$. burnetii shedding in dairy goat herds and to monitor a $\mathrm{Q}$ fever outbreak. Repeated vaccination was an effective method to reduce the number of C. burnetii BTM PCR-positive farms. On farms where pregnant goats were culled, there was an increased risk of becoming BTM PCR positive. This increased risk was probably caused by presence of chronically infected and intermittently shedding goats that were nonpregnant at the time of culling and persisted on those farms. Because several measures to prevent environmental contamination were implemented at the same time, it was not possible to measure the effect of each particular measure separately. The total package of implemented measures has most probably caused the end of the human Q fever outbreak in the Netherlands (Dijkstra et al., 2012).

\section{ACKNOWLEDGMENTS}

This study was financially supported by the Dutch Ministry of Economic Affairs (EZ; The Hague, the Netherlands). We thank our GD Animal Health colleagues Gerdien van Schaik and Wim Swart for their suggestions on the data analysis. We also thank the Dutch Enterprise Agency (RVO; Assen, the Netherlands) and the Dutch Food and Consumer Product Safety Authority (NVWA; Utrecht, the Netherlands) for providing the data on vaccination and culling.

\section{REFERENCES}

Arricau-Bouvery, N., and A. Rodolakis. 2005. Is Q fever an emerging or re-emerging zoonosis? Vet. Res. 36:327-349.

Arricau Bouvery, N., A. Souriau, P. Lechopier, and A. Rodolakis. 2003. Experimental Coxiella burnetii infection in pregnant goats: Excretion routes. Vet. Res. 34:423-433.

Babudieri, B., and C. Moscovici. 1952. Experimental and natural infections of birds by Coxiella burnetii. Nature 169:195-196. 
Berri, M., E. Rousset, J. L. Champion, P. Russo, and A. Rodolakis. 2007. Goats may experience reproductive failures and shed Coxiella burnetii at two successive parturitions after a $\mathrm{Q}$ fever infection. Res. Vet. Sci. 83:47-52.

Boarbi, S., M. Mori, E. Rousset, K. Sidi-boumedine, M. Van Esbroeck, and D. Fretin. 2014. Prevalence and molecular typing of Coxiella burnetii in bulk tank milk in Belgian dairy goats, 2009-2013. Vet. Microbiol. 170:117-124.

Dijkstra, F., W. van der Hoek, N. Wijers, B. Schimmer, A. Rietveld, C. J. Wijkmans, P. Vellema, and P. M. Schneeberger. 2012. The 2007-2010 Q fever epidemic in the Netherlands: Characteristics of notified acute $\mathrm{Q}$ fever patients and the association with dairy goat farming. FEMS Immunol. Med. Microbiol. 64:3-12.

Fretz, R., W. Schaeren, M. Tanner, and A. Baumgartner. 2007. Screening of various foodstuffs for occurrence of Coxiella burnetii in Switzerland. Int. J. Food Microbiol. 116:414-418.

García-Pérez, A. L., I. Astobiza, J. F. Barandika, R. Atxaerandio, A. Hurtado, and R. A. Juste. 2009. Short communication: Investigation of Coxiella burnetii occurrence in dairy sheep flocks by bulktank milk analysis and antibody level determination. J. Dairy Sci. 92:1581-1584.

Guatteo, R., F. Beaudeau, A. Joly, and H. Seegers. 2007. Coxiella burnetii shedding by dairy cows. Vet. Res. 38:849-860.

Hermans, M. H. A., C. J. J. Huijsmans, J. A. Schellekens, P. H. M. Savelkoul, and P. C. Wever. 2011. Coxiella burnetii DNA in goat milk after vaccination with Coxevac. Vaccine 29:2653-2656.

Hesselink, J. W. 1993. Incidence of hydrometra in dairy goats. Vet. Rec. 132:110-112.

Hogerwerf, L., R. Van den Brom, H. I. Roest, A. Bouma, P. Vellema, M. Pieterse, D. Dercksen, and M. Nielen. 2011. Reduction of Coxiella burnetii prevalence by vaccination of goats and sheep, the Netherlands. Emerg. Infect. Dis. 17:379-386.

Kampen, A. H., P. Hopp, G. M. Grøneng, I. Melkild, A. M. Urdahl, A. C. Karlsson, and J. Tharaldsen. 2012. No indication of Coxiella burnetii infection in Norwegian farmed ruminants. BMC Vet. Res. 8:59.

Livingstone, M., N. Wheelhouse, S. W. Maley, and D. Longbottom. 2009. Molecular detection of Chlamydophila abortus in post-abortion sheep at oestrus and subsequent lambing. Vet. Microbiol. 135:134-141.

Longbottom, D., M. Livingstone, S. Maley, A. van der Zon, M. Rocchi, K. Wilson, N. Wheelhouse, M. Dagleish, K. Aitchison, S. Wattegedera, M. Nath, G. Entrican, and D. Buxton. 2013. Intranasal infection with Chlamydia abortus induces dose-dependentlatency and abortion in sheep. PLoS ONE 8:e57950.

Papp, J. R., P. E. Shewen, and C. J. Gartley. 1994. Abortion and subsequent excretion of Chlamydiae from the reproductive tract of sheep during estrus. Infect. Immun. 62:3786-3792.

Rahimi, E., M. Ameri, G. Karim, and A. Doosti. 2011. Prevalence of Coxiella burnetii in bulk milk samples from dairy bovine, ovine, caprine, and camel herds in Iran as determined bypolymerase chain reaction. Foodborne Pathog. Dis. 8:307-310.

Rodolakis, A., M. Berri, C. Héchard, C. Caudron, A. Souriau, C. C. Bodier, B. Blanchard, P. Camuset, P. Devillechaise, J. C. Natorp, J. P. Vadet, and N. Arricau-Boevery. 2007. Comparison of Coxiella burnetii shedding in milk of dairy bovine, caprine, and ovine herds. J. Dairy Sci. 90:5352-5360.

Roest, H. J., B. van Gelderen, A. Dinkla, D. Frangoulidis, F. G. van Zijderveld, J. Rebel, and L. van Keulen. 2012. Q fever in pregnant goats: Pathogenesis and excretion of Coxiella burnetii. PLoS ONE 7:e48949.

Sánchez, J., A. Souriau, A. J. Buendia, N. Arricau-Bouvery, C. M. Martinez, J. Salinas, A. Rodolakis, and J. A. Navarro. 2006. Experimental Coxiella burnetii infection in pregnant goats: a histopathological and immunohistochemical study. J. Comp. Pathol. 135:108-115.

Sejian, V., and R. S. Srivastava. 2010. Pineal-adrenal-immune system relationship under thermal stress: effect on physiological, endocrine, and non-specific immune response in goats. J. Physiol. Biochem. 66:339-349.

Van den Brom, R., K. Lievaart-Peterson, S. Luttikholt, K. Peperkamp, W. Wouda, and P. Vellema. 2012a. Abortion in small ruminants in the Netherlands between 2006 and 2011. Tijdschr. Diergeneeskd. 137:450-457.

Van den Brom, R., L. Moll, G. van Schaik, and P. Vellema. 2013a. Demography of $\mathrm{Q}$ fever seroprevalence in sheep and goats in the Netherlands in 2008. Prev. Vet. Med. 109:76-82.

Van den Brom, R., E. van Engelen, S. Luttikholt, L. Moll, K. van Maanen, and P. Vellema. 2012b. Coxiella burnetii in bulk tank milk samples from dairy goat and dairy sheep farms in the Netherlands in 2008. Vet. Rec. 170:310

Van den Brom, R., E. van Engelen, J. Vos, S. J. M. Luttikholt, L. Moll, H. I. J. Roest, H. M. J. F. van der Heijden, and P. Vellema. 2013b. Detection of Coxiella burnetii in the bulk tank milk from a farm with vaccinated goats, by using a specific PCR technique. Small Rumin. Res. 110:150-154.

Van den Brom, R., and P. Vellema. 2009. Q fever outbreaks in small ruminants and people in the Netherlands. Small Rumin. Res. 86:74-79.

van der Hoek, W., J. Hunink, P. Vellema, and P. Droogers. 2011. Q fever in the Netherlands: The role of local environmental conditions. Int. J. Environ. Health Res. 21:441-451.

van der Hoek, W., J. van de Kassteele, B. Bom, A. de Bruin, F. Dijkstra, B. Schimmer, P. Vellema, R. ter Schegget, and P. M. Schneeberger. 2012. Smooth incidence maps give valuable insight into $\mathrm{Q}$ fever outbreaks in The Netherlands. Geospat. Health 7:127-134.

van Engelen, E., S. J. M. Luttikholt, N. H. M. T. Peperkamp, P. Vellema, and R. Van den Brom. 2014. Small ruminant abortions in the Netherlands during lambing season 2012- 2013. Vet. Rec. 174:506.

Van Steenbergen, J. E., G. Morroy, C. A. R. Groot, F. G. H. Ruikes, J. H. Marcelis, and P. Speelman. 2007. An outbreak of Q fever in the Netherlands-Possible link to goats (article in Dutch). Ned. Tijdschr. Geneeskd. 151:1998-2003.

Vellema, P., L. Moll, R. Van den Brom, and D. Dercksen. 2010a. Research into possible side effects of Coxevac. GD Animal Health Report 2080024. GD Animal Health, Deventer, the Netherlands.

Vellema, P., H. J. Roest, A. van der Spek, R. Ruuls, A. Dinkla, D. Dercksen, and L. Moll. 2010b. An investigation into the possibility of shedding of Coxiella burnetii after vaccination with Coxevac. GD Animal Health Report 2080032. GD Animal Health, Deventer, the Netherlands.

Vellema, P., and R. Van den Brom. 2014. The rise and control of the 2007-2012 human Q fever outbreak in the Netherlands. Small Rumin. Res. 118:69-78.

Wouda, W., and D. P. Dercksen. 2007. Abortion and stillbirth among dairy goats as a consequence of Coxiella burnetii (article in Dutch). Tijdschr. Diergeneeskd. 132:908-911. 